\title{
Ann Arbor Stage IIIE and IIIS
}

National Cancer Institute

\section{Source}

National Cancer Institute. Ann Arbor Stage IIIE and IIIS. NCI Thesaurus. Code C150551.

An Ann Arbor classification lymphoma stage term that refers to extralymphatic organ or site involvement plus involvement of lymph nodes on both sides of the diaphragm or involvement of lymph nodes on both sides of the diaphragm plus involvement of the spleen. 